Revista de Matemática: Teoría y Aplicaciones 1994 1(1) : 61-71

CIMPA - UCR - CCSS ISSN: 1409-2433

\title{
ESTUDIO CRÍTICO DEL MODELO DE MANLY-PARR
}

\author{
Jorge Poltronieri VArgas*
}

\begin{abstract}
Resumen
Manly y Parr (1968) publican un modelo para estimar los parámetros de una población animal. Sin embargo el modelo presenta una incongruencia en cuanto a la especificación de una clase de individuos en la población. En el artículo se elimina este problema. También se comparan los estimadores con los de Jolly-Seber y se dan las fórmulas de varianza-covarianza de los estimadores.
\end{abstract}

\begin{abstract}
Manly and Parr in 1968 obtain a model which give an estimation of the parameters of an animal population. The model presents some weakness on the specifications of the class of individuals in the population. In the present paper we solve this problem in the model. We compare the estimators in the new model with the Jolly-Seber and we give the formulae of variance-covariance of the estimators.
\end{abstract}

\section{Introducción}

En biología, el problema de estimar los parámetros de una población animal (tamaño, tasa de mortalidad, tasa de natalidad, etc.) es muy importante, pues es su conocimiento lo que permite estudiar la dinámica de la población considerada.

Cuando la observación directa es imposible, es a partir de muestras sucesivas que estos parámetros son estimados, y por esto los modelos de captura-recaptura se han desarrollado. La técnica utilizada consiste en tomar $k$ muestras aleatorias $n_{1}, \ldots, n_{k}$. Los individuos de cada muestra son marcados y devueltos a la población, antes de proceder a un nuevo muestreo.

En 1968 Manly y Parr proponen un modelo simple para estimar la talla $N_{i}$ de una población animal, por el método de captura-recaptura, sin hacer hipótesis sobre la probabilidad de sobrevivencia. Manly (1969) da las varianzas de los estimadores. Sin embargo este modelo presenta una incongruencia en cuanto a la especificación de una clase de individuos en la población. Aquí proponemos una modificación del modelo que elimina esta dificultad teórica. Es el objeto de este artículo.

\footnotetext{
*Escuela de Matemática, Universidad de Costa Rica, 2060 San José, Costa Rica. E-Mail: jpvargas@racsa.co.cr
} 


\section{El modelo de Manly-Parr: hipótesis}

- Se considera que todo individuo en la población tiene la misma probabilidad $p_{i}(=$ $1-q_{i}$ ) de captura al instante $t_{i}$, independientemente de su historial de captura.

- Se supone que se conoce una clase de individuos $X_{i}$ en la población que están presentes al instante $t_{i}^{-}$.

- Todo individuo en la población tiene la misma probabilidad $\theta_{i}$ de estar en la clase $X_{i}$.

- La marca y la captura no afectan la conducta de los individuos.

- El tiempo transcurrido de muestreo, es pequeño con respecto al tiempo transcurrido entre dos muestras sucesivas.

\section{Notación y definiciones:}

$k$ : Número de muestras. Para todo lo que sigue $i$ es tal que $1 \leq i \leq k$

$N_{i}$ : Tamaño de la población al instante $t_{i}^{-}$

$n_{i}$ : Número de capturas al instante $t_{i}$

$X_{i}$ : (Abusando de la escritura) tamaño de la clase $X_{i}$ en $t_{i}^{-}$

$x_{i}$ : Número de individuos capturados de $X_{i}$ al instante $t_{i}$

$R_{i}$ : Número de individuos devueltos a la población al terminar la muestra $i$

$\phi_{i}$ : Probabilidad de sobrevivencia de la población entre los instantes $t_{i}^{+}$y $t_{i+1}^{-}$

$B_{i}$ : Número de individuos nuevos en la población entre los instantes $t_{i}^{+}$y $t_{i+1}^{-}$

$s_{i}: n_{i}-x_{i}$.

Se denotará por $\hat{V}_{i}$ un estimador del parámetro $V_{i}$ de la población, por $n_{i}, x_{i}, X_{i}, s_{i}$ las realizaciones de las variables aleatorias ${ }_{i, i}, i, \mathbf{s}_{i}$ respectivamente y por $E(\mathbf{t})=\bar{t}$, la esperanza de la variable aleatoria $\mathbf{t}$.

\section{Formalización del modelo}

A cada instante $t_{i} \quad(1 \leq i \leq k)$ se capturan $n_{i}\left(=x_{i}+s_{i}\right)$ individuos entre la población de talla $N_{i}$ (parámetro fijo). La situación de captura en $t_{i}$ se ilustra en la tabla siguiente:

\begin{tabular}{c|c|c|c} 
& capturados en $t_{i}$ & no capturados en $t_{i}$ & total \\
\hline Dentro de $X_{i}$ & $x_{i}$ & $X_{i}-x_{i}$ & $X_{i}$ \\
\hline Fuera de $X_{i}$ & $s_{i}$ & $N_{i}-X_{i}-s_{i}$ & $N_{i}-X_{i}$ \\
\hline Total & $n_{i}$ & $N_{i}-n_{i}$ & $N_{i}$ \\
\hline
\end{tabular}


Si la probabilidad de captura es independiente de la pertenencia o no a la clase $X_{i}$, la ley de distribución de las variables aleatorias ${ }_{i},{ }_{i}-{ }_{i}, \mathbf{s}_{i}, N_{i}-{ }_{i}-\mathbf{s}_{i}$ condicionada a $N_{i}$ es una multinomial con 4 categorías de probabilidades respectivas $\theta_{i} p_{i}, \theta_{i}\left(1-p_{i}\right), p_{i}\left(1-\theta_{i}\right),(1-$ $\left.p_{i}\right)\left(1-\theta_{i}\right)$. La distribución es de la forma:

$$
\begin{gathered}
P\left({ }_{i}=x_{i, i}-{ }_{i}=X_{i}-x_{i}, \mathbf{s}_{i}=s_{i}, N_{i}-{ }_{i}-\mathbf{s}_{i}=N_{i}-X_{i}-s_{i}\right)= \\
\frac{N_{i} !}{x_{i} !\left(X_{i}-x_{i}\right) ! s_{i} !\left(N_{i}-X_{i}-s_{i}\right) !} p_{i}^{n_{i}}\left(1-p_{i}\right)^{N_{i}-n_{i}} \theta_{i}^{X_{i}}\left(1-\theta_{i}\right)^{N_{i}-X_{i}} .
\end{gathered}
$$

\subsection{Estimación por el método de máxima verosimilitud}

Las ecuaciones de máxima verosimilitud nos dan:

$$
\begin{gathered}
\hat{p}_{i}={ }_{i} / i \\
\hat{N}_{i}={ }_{i} / \hat{p}_{i}=\frac{i}{{ }_{i}}{ }_{i}{ }_{i}+\frac{\mathbf{s}_{i}}{{ }_{i}}{ }_{i} \\
\hat{\theta}_{i}={ }_{i} / \hat{N}_{i} .
\end{gathered}
$$

El argumento más fuerte en favor de (3) es que pocas hipótesis se hacen sobre la conducta de los individuos. No es necesario, en principio, suponer que la probabilidad de sobrevivencia

es independiente de la edad de los individuos. Estas hipótesis son necesarias para todos los otros modelos de captura-recaptura.

\subsection{Varianzas de los estimadores}

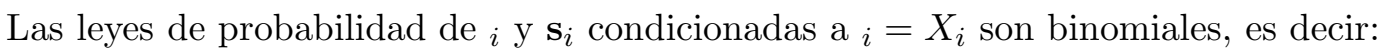

$$
\begin{gathered}
P\left({ }_{i}=x_{i} / X_{i}\right)=\left(\begin{array}{c}
X_{i} \\
x_{i}
\end{array}\right) p_{i}^{x_{i}}\left(1-p_{i}\right)^{X_{i}-x_{i}} \\
P\left(\mathbf{s}_{i}=s_{i} / X_{i}\right)=\left(\begin{array}{c}
N_{i}-X_{i} \\
s_{i}
\end{array}\right) p_{i}^{s_{i}}\left(1-p_{i}\right)^{N_{i}-X_{i}-s_{i}}
\end{gathered}
$$

por lo que:

$$
{ }_{i}=X_{i} p_{i}+\delta x_{i} \quad \mathrm{y} \quad \mathbf{s}_{i}=\left(N_{i}-X_{i}\right) p_{i}+\delta s_{i}
$$

con:

$$
\begin{array}{ll}
E\left(\delta x_{i} / X_{i}\right)=0 & E\left(\delta x_{i}^{2} / X_{i}\right)=X_{i} p_{i}\left(1-p_{i}\right) \\
E\left(\delta s_{i} / X_{i}\right)=0 & E\left(\delta s_{i}^{2} / X_{i}\right)=\left(N_{i}-X_{i}\right) p_{i}\left(1-p_{i}\right) .
\end{array}
$$

El desarrollo de Taylor para $N_{i}$ condicionada a $X_{i}$ da:

$$
\hat{N}_{i}-N_{i} \simeq\left(N_{i}-X_{i}\right)\left(\frac{\delta s_{i}}{s_{i}}-\frac{\delta x_{i}}{x_{i}}-\frac{\delta x_{i} \delta s_{i}}{s_{i} x_{i}}+\frac{\delta x_{i}^{2}}{x_{i}^{2}}+\frac{\delta s_{i} \delta x_{i}^{2}}{s_{i} x_{i}^{2}}\right) .
$$


Si se toma la esperanza condicionada $\mathrm{a}_{i}=X_{i}$, se obtiene asintóticamente:

$$
E\left(\hat{N}_{i} / X_{i}\right)=N_{i}+\left(N_{i} / X_{i}-1\right)\left(1 / p_{i}-1\right)
$$

La distribución de ${ }_{i}$ condicionada a $N_{i}$ es binomial:

$$
P\left({ }_{i}=X_{i}\right)=\left(\begin{array}{c}
N_{i} \\
X_{i}
\end{array}\right) \theta_{i}^{X_{i}}\left(1-\theta_{i}\right)^{N_{i}-X_{i}}
$$

por lo que:

$$
{ }_{i}=N_{i} \theta_{i}+\delta X_{i} \quad \text { con } \quad E\left(\delta X_{i}\right)=0 \quad \text { y } \quad E\left(\delta X_{i}^{2}\right)=N_{i} \theta_{i}\left(1-\theta_{i}\right) .
$$

La ecuación (5) se escribe (asintóticamente):

$$
E\left(\hat{N}_{i}-N_{i} / X_{i}\right)=\left(1 / \theta_{i}-1-\frac{\delta X_{i}}{N_{i} \theta_{i}^{2}}+\frac{\delta X_{i}^{2}}{N_{i}^{2} \theta_{i}^{3}}\right)\left(1 / p_{i}-1\right) .
$$

Si se toma la esperanza sobre ${ }_{i}$ se tiene:

$$
E\left(\hat{N}_{i}\right)=N_{i}+\left(1 / \theta_{i}-1\right)\left(1 / p_{i}-1\right) .
$$

Se puede estudiar el estimador modificado:

$$
\begin{aligned}
N_{i}^{*} & =\frac{(i+1)\left({ }_{i}+1\right)}{i+1}-1 \\
& \simeq \frac{i i}{i}\left(1+\frac{1}{i}\right)\left(1+\frac{1}{i}\right)\left(1-\frac{1}{i}\right)-1 \\
& \simeq \frac{i i}{i}-\left(1-\frac{i}{i}\right)\left(1-\frac{i}{i}\right)
\end{aligned}
$$

y tomando la esperanza condicionada a ${ }_{i}$ y después la esperanza sobre ${ }_{i}$ se tiene:

$$
E\left(N_{i}^{*}\right) \simeq E\left(\hat{N}_{i}\right)-\left(1 / p_{i}-1\right)\left(1 / \theta_{i}-1\right) \simeq N_{i}
$$

Utilizando la misma técnica se obtiene la varianza asintótica de $\hat{N}_{i}$ condicionada a ${ }_{i}$ :

$$
\operatorname{Var}\left(\hat{N}_{i} / X_{i}\right)=N_{i}\left(1 / p_{i}-1\right)\left(N_{i} / X_{i}-1\right)
$$

y después la esperanza sobre ${ }_{i}$ y siempre en primera aproximación:

$$
\begin{gathered}
\operatorname{Var}\left(\hat{N}_{i}\right)=N_{i}\left(1 / p_{i}-1\right)\left(1 / \theta_{i}-1\right) \\
\operatorname{cov}\left(\hat{N}_{i}, \hat{N}_{j}\right)=0 \quad i \neq j .
\end{gathered}
$$




\subsection{Especificación de la clase $X_{i}$}

Vamos ahora a precisar el contenido de la clase de $X_{i}$ para una población abierta, en la cual la emigración es permanente (caso considerado por el modelo de Jolly-Seber) y donde no hay muerte sobre captura $\left(R_{i}=n_{i}\right)$.

Sea $r_{i}^{\prime}$ el número de individuos marcados capturados en $t_{i}$ y recapturados después de $t_{i}$. Se define $X_{i}=r_{i}^{\prime}+Z_{i}$, donde $Z_{i}$ es el número de individuos marcados no capturados en $t_{i}$ y recapturados después de $t_{i}$, es decir, $X_{i}$ es el número de individuos marcados capturados antes y después de $t_{i}$, y $x_{i}=r_{i}^{\prime}$.

La probabilidad de captura $p_{i}$ es estimada por:

$$
\hat{p}_{i}=\mathbf{r}_{i}^{\prime} /\left(\mathbf{r}_{i}^{\prime}+{ }_{i}\right)
$$

Comparemos este estimador con el de Jolly- Seber (los estimadores de Jolly-Seber se denotarán con $\sim$ ).

$$
\tilde{p}_{i}=\frac{i}{\tilde{M}_{i}}=\frac{\mathbf{r}_{i} \frac{i}{i}}{\mathbf{r}_{i} \frac{i}{i}+{ }_{i}}
$$

donde $m_{i}$ es el número de individuos marcados capturados en $t_{i}, r_{i}$ es el número de individuos caturados en $t_{i}$, devueltos a la población y recapturados después de $t_{i}, \tilde{M}_{i}$ es el estimador de la población marcada presente al instante $t_{i}$. El estimador $\tilde{M}_{i}={ }_{i}+\frac{\mathbf{r}_{i}}{}$.

Cuando $\phi_{i}$ es constante para todos los individuos $r_{i}^{\prime} / r_{i} \simeq m_{i} / n_{i}$ y los dos estimadores tienen valores similares. $\mathrm{Si} \phi_{i}$ no es constante y depende de la edad por ejemplo, $\tilde{p}_{i}$ puede no ser válido si las estructuras de edad de la población y de los capturados difieren de manera importante. Esta situación se da si la probabilidad de captura depende de la edad.

Para los datos de la Tabla 1, las estimaciones del modelo de Manly-Parr están dadas en la Tabla 2, y comparadas con las de Jolly-Seber. No hay gran diferencia entre los grupos de estimadores.

\begin{tabular}{|c|c|c|c|c|}
$i$ & $n_{i}$ & $r_{i}^{\prime}=x_{i}$ & $Z_{i}$ & $X_{i}$ \\
\hline 1 & 57 & 0 & 0 & 0 \\
\hline 2 & 52 & 15 & 11 & 26 \\
\hline 3 & 52 & 14 & 12 & 26 \\
\hline 4 & 31 & 10 & 20 & 30 \\
\hline 5 & 54 & - & - & - \\
\hline
\end{tabular}

Tabla 1: Manly- Parr (1968) 


\begin{tabular}{c|ccccc||ccccc}
\hline & \multirow{2}{*}{$\hat{p}_{i}$} & $\hat{N}_{i}$ & $\operatorname{Var}\left(\hat{N}_{i}\right)^{\frac{1}{2}}$ & $\hat{\phi}_{i}$ & $\hat{B}_{i}$ & $\tilde{p}_{i}$ & $\tilde{N}_{i}$ & $\operatorname{Var}\left(\tilde{N}_{i}\right)^{\frac{1}{2}}$ & $\tilde{\phi}_{i}$ & $\tilde{B}_{i}$ \\
& & & & & & & & & & \\
\hline 1 & - & - & - & .76 & - & - & - & - & .78 & - \\
2 & .5769 & 90.13 & 12.8 & .71 & 32 & .5590 & 93.03 & 16.3 & .74 & 30 \\
3 & .5385 & 96.56 & 15.0 & .69 & 26 & .5287 & 98.35 & 14.2 & .76 & 32 \\
4 & .3333 & 93.01 & 19.8 & - & - & .2914 & 106.37 & 23.6 & - & - \\
5 & - & - & - & - & - & - & - & - & - & - \\
\hline
\end{tabular}

Tabla 2: Manly-Parr (1968)

\subsection{Estimación de la sobrevivencia}

Supongamos que $\phi_{i} n_{i}$ individuos devueltos a la población en $t_{i}^{+}$han sobrevivido al instante $t_{i+1}^{-}$y sea $n_{i, i+1}$ el número de estos individuos capturados en $t_{i+1}$. Siempre bajo la hipótesis del modelo:

$$
E\left({ }_{i, i+1} / \phi_{i} n_{i}\right)=\phi_{i} \bar{n}_{i} p_{i+1}
$$

y $\phi_{i}$ es estimado por:

$$
\hat{\phi}_{i}={ }_{i, i+1} /{ }_{i} \hat{p}_{i+1} \text {. }
$$

El estimador de Jolly-Seber para $\phi_{i}$ es:

$$
\tilde{\phi}_{i}=\frac{{ }_{i+1} \frac{\mathbf{r}_{i}}{\mathbf{r}_{i}+i}}{{ }_{i} \tilde{p}_{i+1}} .
$$

Si la probabilidad de sobrevivencia es independiente de la marca y de la edad del individuo, entonces $n_{i, i+1} / r_{i}$ es aproximadamente $m_{i+1} /\left(r_{i}+Z_{i}\right)$ y los estimadores dan resultados semejantes.

Un estimador de $B_{i}$, número de individuos nuevos en la población, es (si $\phi_{i}$ es la misma para marcados y no marcados):

$$
\hat{B}_{i}=\hat{N}_{i+1}-\hat{N}_{i} \hat{\phi}_{i}
$$

\section{Crítica del modelo}

Manly y Parr afirman que la diferencia fundamental con Jolly-Seber, es que pocas hipótesis se haen sobre la probabilidad de sobrevivencia. Sin embargo existe una crítica fuerte al modelo: con la escogencia adoptada de $X_{i}$ "la probabilidad $\theta_{i}$ de ser capturado antes y después de $t_{i}$ no es la misma para todos los individuos". Por ejemplo si hay migración entre el instante $t_{i-1}$ y $t_{i}$ un inmigrante tiene probabilidad nula de pertenecer a la clase $X_{i}$. También observamos que para que un individuo pertenezca a la clase $X_{i}$, debe ser marcado antes de $t_{i}$ y recapturado después de $t_{i}$, lo que implica que las probabilidades 
de sobrevivencia y de captura, deben ser las mismas para todos los individuos. Sin embargo el modelo es válido si los eventos "captura en $t_{i}$ " y "pertenencia a la clase $X_{i}$ " son independientes para cada individuo.

Observemos que si hay muerte en la captura, estos dos eventos son dependientes: un individuo capturado en $t_{i}$ tiene una probabilidad más pequeña de ser recapturado que un individuo no tomado en $t_{i}$.

\subsection{Modificación del modelo de Manly-Parr}

Hemos visto que en la determinación de la clase $X_{i}$ hay una dificultad teórica, pues todo individuo en la población no tiene la misma probabilidad de pertenecer a $X_{i}$. Para resolver este problema se procede de la siguiente manera.

Sea $M_{i}$ la talla de la población de individuos marcados presentes al instante $t_{i}^{-}$. Aplicando la teoría de Manly-Parr a la población marcada se define una clase $L_{i}$ de individuos marcados, presentes al instante $t_{i}^{-}$. Entre estos individuos de la clase $L_{i}$, se capturan $l_{i}$ individuos al instante $t_{i}, m_{i}=l_{i}+v_{i}$, donde $v_{i}$ es el número de individuos marcados capturados en $t_{i}$ que no pertenecen a $L_{i}$. Un estimador para $M_{i}$ es:

$$
\hat{M}_{i}={ }_{i i} / \mathbf{l}_{i}={ }_{i}+\mathbf{v}_{i i} / \mathbf{l}_{i}
$$

Así cuando se define $L_{i}=r_{i}^{\prime}+Z_{i}\left(l_{i}=r_{i}^{\prime}\right)$ la crítica al modelo no es válida.

Las leyes de probabilidad de $\mathbf{l}_{i} \mathrm{y}_{\mathbf{v}_{i}}$ condicionada $\mathrm{a}_{i}=L_{i}$ son binomiales y:

$$
\begin{gathered}
E\left(\hat{M}_{i} / L_{i}\right)=M_{i}+\left(1-M_{i} / L_{i}\right)\left(1-1 / p_{i}\right) \\
\operatorname{Var}\left(\hat{M}_{i} / L_{i}\right)=\left(M_{i}-L_{i}\right)\left(1 / p_{i}-1\right) M_{i} / L_{i} .
\end{gathered}
$$

Sea $\psi_{i}$ la probabilidad de un individuo de $M_{i}$ de ser incluido en $L_{i}\left(\psi_{i}=L_{i} / M_{i}\right)$. Sabemos que:

$$
\begin{gathered}
P\left({ }_{i}=L_{i} / M_{i}\right)=\left(\begin{array}{c}
M_{i} \\
L_{i}
\end{array}\right) \psi_{i}^{L_{i}}\left(1-\psi_{i}\right)^{M_{i}-L_{i}} \\
E\left(\delta L_{i}\right)=0 \quad \text { y } \quad E\left(\delta L_{i}^{2}\right)=M_{i} \psi_{i}\left(1-\psi_{i}\right) .
\end{gathered}
$$

Si tomamos la esperanza sobre ${ }_{i}$ en (11) y (12), se tiene asintóticamente:

$$
\begin{gathered}
E\left(\hat{M}_{i}-M_{i}\right)=\left(1 / \psi_{i}-1\right)\left(1 / p_{i}-1\right)=\left(M_{i}-\bar{m}_{i}\right)\left(1 / \bar{l}_{i}-1 / \bar{m}_{i}\right) \\
\operatorname{Var}\left(\hat{M}_{i}\right)=M_{i}\left(1 / \psi_{i}-1\right)\left(1 / p_{i}-1\right)=M_{i}\left(M_{i}-\bar{m}_{i}\right)\left(1 / \bar{l}_{i}-1 / \bar{m}_{i}\right) \\
\operatorname{cov}\left(\hat{M}_{i}, \hat{M}_{j}\right)=0 \quad i \neq j .
\end{gathered}
$$

Observemos que:

$$
\hat{p}_{i}=\mathbf{l}_{i} / i \quad \hat{\psi}_{i}={ }_{i} / \hat{M}_{i} \quad \hat{M}_{i}={ }_{i} / \hat{p}_{i}
$$

son los estimadores de máxima verosimilitud. 


\subsection{Estudio del modelo modificado}

De manera general la modificación del modelo presenta ventajas:

- Se supone solamente la existencia de una subpoblación en la cual todos los individuos tienen la misma probabilidad de pertenecer a la clase $L_{i}$.

- La hipótesis "no hay muertes sobre captura" es solamente para esta subpoblación.

Estas ventajas nos colocan en una situación más realista.

Sea $W_{i}$ esta subpoblación. Sea $w_{i}$ el número de individuos de $W_{i}$ capturados al instante $t_{i}$

$$
U_{i}=N_{i}-W_{i}, \quad u_{i}=n_{i}-w_{i} .
$$

La ley de probabilidad para esta situación, es un producto de leyes multinomiales, es decir:

$$
\begin{gathered}
P\left(\mathbf{u}_{i}=u_{i}, \mathbf{l}_{i}=l_{i},_{i}-\mathbf{l}_{i}=L_{i}-l_{i}, \mathbf{v}_{i}=v_{i} / N_{i}, W_{i}\right)= \\
P\left(\mathbf{u}_{i}=u_{i} / N_{i}, W_{i}\right) P\left(\mathbf{l}_{i}=l_{i},{ }_{i}-\mathbf{l}_{i}=L_{i}-l_{i}, \mathbf{v}_{i}=v_{i} / N_{i}, W_{i}, u_{i}\right)= \\
\frac{U_{i} !}{\left(U_{i}-u_{i}\right) ! u_{i} !} p_{i}^{u_{i}}\left(1-p_{i}\right)^{U_{i}-u_{i}} \frac{W_{i} !}{l_{i} !\left(L_{i}-l_{i}\right) ! v_{i} !\left(W_{i}-L_{i}-v_{i}\right) !}\left(\psi_{i} p_{i}\right)^{l_{i}} \times \\
\left(\psi_{i}\left(1-p_{i}\right)\right)^{L_{i}-l_{i}}\left(\left(1-\psi_{i}\right) p_{i}\right)^{v_{i}}\left(\left(1-\psi_{i}\right)\left(1-p_{i}\right)\right)^{W_{i}-L_{i}-v_{i}} \\
=\frac{U_{i} ! W_{i} !}{u_{i} ! l_{i} !\left(L_{i}-l_{i}\right) ! v_{i} !\left(W_{i}-L_{i}-v_{i}\right) !\left(U_{i}-u_{i}\right) !} p_{i}^{n_{i}}\left(1-p_{i}\right)^{N_{i}-n_{i}} \psi_{i}^{L_{i}}\left(1-\psi_{i}\right)^{W_{i}-L_{i}}
\end{gathered}
$$

Como la teoría de Manly-Parr es válida para $W_{i}$, todo el razonamiento puede aplicarse a $W_{i}$. Las formulas (5), (6), (7) y (8) no son válidas pues se tiene una clase $L_{i}$ en la población $W_{i}$. Se define un estimador de $N_{i}$ por (si todo individuo en la población $N_{i}$ riene la misma probabilidad $p_{i}$ de ser capturado al instante $t_{i}$ ):

$$
\hat{N}_{i}=\frac{i}{i} \hat{W}_{i}
$$

Se observa que (15) no es el mismo estimador que (3), pues $X_{i}$ y $L_{i}$ están definidas de manera diferente. Esta observación indica el error contenido en la definición de la clase $X_{i}$ en el modelo de Manly-Parr, pues: $X_{i}$ es una clase en la población entera, y $L_{i}$ es una clase en la población $W_{i}$. Por otro lado si se define $W_{i}=M_{i}$ y la clase $L_{i}$ por $L_{i}=r_{i}^{\prime}+Z_{i}, l_{i}=r_{i}^{\prime}$, la crítica del modelo no es válida aquí pues todo individuo marcado tiene la misma probabilidad de ser recapturado, i.e. de pertenecer a $L_{i}$, bajo las hipótesis: no hay muerte sobre captura en $M_{i}$, todo individuo en $M_{i}$ tiene la misma probabilidad de sobrevivencia y la probabilidad de captura en $M_{i}$ es la misma en todos los individuos. Si hay muerte sobre captura en los individuos nuevos, la probabilidad de ser devueltos a la 
población va a estar ligado a la marca. Pero este fenómeno no presenta problema en la teoría. En este caso $\nu_{i}^{\prime}$ (probabilidad de ser devuelto a la población para la población marcada) vale 1. La probabilidad de ser devuelto a la población para los individuos no marcados es:

$$
\hat{\nu}_{i}={ }^{\circ}
$$

$i^{-}{ }_{i} \overline{i-i}$.

La condición: "no hay muerte sobre captura en $M_{i}$ (población marcada)" es una consecuencia de la definición que se adoptó de $L_{i}$, pues puede existir otra definición de $L_{i}$, donde la muerte sobre captura no afecte $\psi_{i}$. Por ejemplo, si a cada instante $t_{i}$ se conoce el número de individuos marcados en la población, se define $W_{i}=M_{i}, L_{i}=M_{i}\left(l_{i}=m_{i}\right)$ y la muerte sobre captura en $M_{i}$ no afecta la probabilidad de pertenecer a $L_{i}$ (que vale 1 ).

Observemos que si define $X_{i}=M_{i}, \theta_{i}=X_{i} / N_{i}$ no es la misma para todos los individuos de la población en el caso en que hay inmigración.

Un estimador de la probabilidad $\phi_{i}$ de sobrevivencia entre los instantes $t_{i}$ y $t_{i+1}$ está dado por el cociente:

$$
\hat{\phi}_{i}=\hat{M}_{i+1} /\left(\hat{M}_{i}-{ }_{i}+{ }_{i}\right)
$$

Bajo la hipótesis que todo individuo en la población tiene la misma probabilidad de sobrevivencia, el número de individuos nuevos $B_{i}$ en la población entre $t_{i}$ y $t_{i+1}$, se estima por:

$$
\hat{B}_{i}=\hat{N}_{i+1}-\hat{\phi}\left(\hat{N}_{i}-{ }_{i}+{ }_{i}\right)
$$

\subsection{Varianzas y covarianzas del modelo modificado}

Sabemos que:

$$
\begin{gathered}
E\left(\hat{M}_{i}\right)=M_{i}+\left(1 / \psi_{i}-1\right)\left(1 / p_{i}-1\right)=M_{i}+\left(M_{i}-\bar{m}_{i}\right)\left(1 / \bar{l}_{i}-1 / \bar{m}_{i}\right) \\
\operatorname{Var}\left(\hat{M}_{i}\right)=M_{i}\left(1 / \psi_{i}-1\right)\left(1 / p_{i}-1\right)=M_{i}\left(M_{i}-\bar{m}_{i}\right)\left(1 / \bar{l}_{i}-1 / \bar{m}_{i}\right) .
\end{gathered}
$$

Utilizando el desarrollo de Taylor a partir de las fórmulas (15), (16) y (17), se tienen las varianzas y covarianzas de los estimadores $\hat{N}_{i}, \hat{\phi}_{i}$ y $\hat{B}_{i}$ :

$$
\begin{gathered}
E\left(\hat{N}_{i}\right)=N_{i}+\rho_{i}^{-1}\left(1 / \psi_{i}-1\right)\left(1 / p_{i}-1\right)=N_{i}+\left(N_{i}-\bar{n}_{i}\right)\left(1 / \bar{l}_{i}-1 / \bar{m}_{i}\right) \\
\operatorname{Var}\left(\hat{N}_{i}\right)=N_{i}\left(N_{i}-\bar{n}_{i}\right)\left(1 / \bar{l}_{i}-1 / \bar{n}_{i}\right) \\
\operatorname{cov}\left(\hat{N}_{i}, \hat{N}_{j}\right)=0 \quad i \neq j \\
\operatorname{Var}\left(\hat{\phi}_{i}\right)=\phi_{i}^{2}\left[\frac{\left(M_{i+1}-\bar{m}_{i+1}\right)}{M_{i+1}}\left(1 / \bar{l}_{i+1}-1 / \bar{m}_{i+1}\right)+\frac{M_{i}\left(M_{i}-\bar{m}_{i}\right)}{\left(M_{i}-\bar{m}_{i}+\bar{R}_{i}\right)^{2}}\left(1 / \bar{l}_{i}-1 / \bar{m}_{i}\right)\right]
\end{gathered}
$$




$$
\begin{gathered}
\operatorname{cov}\left(\hat{\phi}_{i-1}, \hat{\phi}_{i}\right)=-\frac{\phi_{i-1} \phi_{i}}{M_{i}-\bar{m}_{i}+\bar{R}_{i}}\left(M_{i}-\bar{m}_{i}\right)\left(1 / \bar{l}_{i}-1 / \bar{m}_{i}\right) \\
\operatorname{cov}\left(\hat{\phi}_{i}, \hat{\phi}_{j}\right)=0 \quad j>i+1 \\
\operatorname{Var}\left(\hat{B}_{i}\right)=\frac{B_{i}^{2}}{M_{i+1}}\left(M_{i}-\bar{m}_{i}\right)\left(1 / \bar{l}_{i}-1 / \bar{m}_{i}\right)+N_{i+1}\left(N_{i+1}-\bar{n}_{i+1}\right) \frac{1-\rho_{i+1}}{\bar{m}_{i+1}} \\
+\phi_{i}^{2} N_{i}\left(N_{i}-\bar{n}_{i}\right) \frac{1-\rho_{i}}{\bar{m}_{i}}+\frac{M_{i}}{\left(M_{i}-\bar{m}_{i}+\bar{R}_{i}\right)^{2}}\left(1 / \bar{l}_{i}-1 / \bar{m}_{i}\right)\left[\phi_{i} \bar{R}_{i}\left(1 / \rho_{i}-1\right)\right]^{2} \\
+\frac{\left(N_{i}-\bar{n}_{i}\right)\left(N_{i+1}-B_{i}\right)\left(1-\rho_{i}\right)\left(1-\phi_{i}\right)}{M_{i}-\bar{m}_{i}+\bar{R}_{i}} \\
\operatorname{cov}\left(\hat{B}_{i-1}, \hat{B}_{i}\right)=\frac{-\phi_{i}\left(N_{i}-\bar{n}_{i}\right)\left(1-\rho_{i}\right)}{\bar{m}_{i}}\left[\frac{B_{i-1} \bar{R}_{i}\left(1 / \psi_{i}-1\right)}{M_{i}-\bar{m}_{i}+\bar{R}_{i}}+N_{i}\right] \\
\operatorname{cov}\left(\hat{B}_{i}, \hat{B}_{j}\right)=0 \quad j>i+1
\end{gathered}
$$

donde $\rho_{i}=\bar{m}_{i} / \bar{n}_{i}$.

Para la expresión de las varianzas y covarianzas en el caso presente se tiene: $W_{i}=M_{i}$, y hay que reemplazar $r_{i}^{\prime}$ por $l_{i}, r_{i}^{\prime}+Z_{i}$ por $L_{i}$ en las formulas (13), (14), (18), (19), (20), (21), (22) y (23). Los estimadores pueden compararsse con los de Jolly-Seber:

$$
\begin{array}{ll}
\hat{p}_{i}=\frac{\mathbf{r}_{i}^{\prime}}{\mathbf{r}_{i}^{\prime}+{ }_{i}} & \tilde{p}_{i}=\frac{\mathbf{r}_{i} \frac{i}{i}}{\mathbf{r}_{i}{ }_{i}-{ }_{i}} \\
\hat{M}_{i}={ }_{i}+\frac{i i}{\mathbf{r}_{i}^{\prime}} & \tilde{M}_{i}=i^{i}+\frac{i i}{\mathbf{r}_{i}} \\
\hat{N}_{i}={ }_{i}+\frac{i i}{\mathbf{r}_{i}^{\prime}} & \tilde{N}_{i}=i_{i}+\frac{i_{i}}{\mathbf{r}_{i i}} .
\end{array}
$$

\section{Conclusión}

Los resultados obtenidos en este trabajo, ayudan a esclarecer la confusión que se establece en el trabajo de Manly y Parr. Es de notar, la similaridad con el modelo de JollySeber, cuando se considera una población abierta (modelo estocástico); caso en que se considera inmigración y emigración. La emigración se supone definitiva, es decir, supone que todo individuo en el momento del muestreo, tiene la misma probabilidad de ser capturado. Así, si un individuo por alguna razón no puede ser observado en el momento de la captura, se considera que no pertenece a la población; situación que puede ser común en la práctica, imponiendo una restricción en la utilización de la técnica. También es importante destacar, que la afirmación no es necesario suponer que la probabilidad de sobrevivencia sea 
independiente de la edad de los individuos, que realizan Manly y Parr, es desacertada. En efecto, en el momento de definir la clase $L_{i}=r_{i}^{\prime}+Z_{i}$, la probabilidad $\phi_{i}\left(=L_{i} / M_{i}\right)$, lleva implícito este hecho.

Una generalización de este modelo, ha sido propuesta en [5]. Este modelo es muy general, y discute el caso particular en que, la marca puede traumatizar al individuo por $l$ períodos en la captura y $q$ períodos en la sobrevivencia. Se estudian los casos $l \leq q$ y $l>q$.

\section{Referencias}

[1] Jolly G. M. (1965). "Explicit estimates from capture-recapture data with both death and inmigrationstochastic model". Biometrika 52, pp. 225-47.

[2] Manly B. F. J. (1969) "Some properties of a method of estimating the size of mobile animal population". Biometrika 56, pp. $407-10$.

[3] Manly B.F.J. - Parr M.J. (1968) "A new method of estimating populations size survivorship and birht rate from capture recapture data". Trans. Soc. Brit. Ent. 18, pp. 81-9.

[4] Poltronieri J. (1977) "Capture-marquage-recapture : Ètude de quelques mèthodes statistiques en cinétique des populations". Thèse Doctorat Université Paul Sabatier, Toulouse, France.

[5] Poltronieri J. (1980) "Modelo con diferentes probabilidades de captura y sobrevivencia". Cienc. Tec. 4(1,2), pp. 37-48.

[6] Seber G.A.F. (1965) “A note on the multiple recapture census". Biometrika 52, pp. 249-59. 